\title{
GLAD!
}

Revue sur le langage, le genre, les sexualités

$07 \mid 2019$

Varia

\section{Le genre tactile : repenser les imbrications entre la matière et la parole au prisme de l'imagination et de l'expérience}

A Tactile Gender: Rethink the Interpenetration of Matter and Discourse through the Lens of Imagination and Experience

\section{Luca Greco}

\section{OpenEdition}

Journals

Édition électronique

URL : http://journals.openedition.org/glad/1714

DOI : $10.4000 /$ glad. 1714

ISSN : 2551-0819

Éditeur

Association GSL

\section{Référence électronique}

Luca Greco, «Le genre tactile : repenser les imbrications entre la matière et la parole au prisme de

l'imagination et de l'expérience », GLAD! [En ligne], 07 | 2019, mis en ligne le 05 décembre 2019,

consulté le 17 décembre 2020. URL : http://journals.openedition.org/glad/1714 ; DOI : https://doi.org/

$10.4000 /$ glad. 1714

Ce document a été généré automatiquement le 17 décembre 2020.

\section{(c) (†) $\odot$}

La revue GLAD! est mise à disposition selon les termes de la Licence Creative Commons Attribution -

Pas d'Utilisation Commerciale - Pas de Modification 4.0 International. 


\title{
Le genre tactile : repenser les imbrications entre la matière et la parole au prisme de l'imagination et de l'expérience
}

\author{
A Tactile Gender: Rethink the Interpenetration of Matter and Discourse through \\ the Lens of Imagination and Experience
}

Luca Greco

\section{Positionnements théoriques}

À partir d'un corpus composé d'enregistrements vidéo effectués dans un atelier drag king à Bruxelles (Greco 2018), d'entretiens produits en Italie avec des féministes ayant participé aux groupes de conscience (gruppi di autocoscienza) dans les années 70, et d'une série de performances artistiques contemporaines, je vais me pencher sur le rôle joué par le toucher dans la construction et la déconstruction du genre dans les interactions ayant lieu dans un contexte militant (ateliers drag king et groupes de conscience féministes) et artistique. En mobilisant les outils théoriques et méthodologiques de l'anthropologie linguistique (C. Goodwin 2017, M.H. Goodwin \& Cekaite 2018), des études de genre (Butler 1993 [2009], Alaimo \& Hekman 2008) et des recherches sur le genre, le langage et la sexualité (Greco 2014, 2015), je vais ouvrir un espace de dialogue possible entre ces trois traditions de recherche. À partir de ce cadre théorique et des questions posées par les chercheurees s'inscrivant dans ces champs disciplinaires, je montrerai comment une prise en compte de la matérialité des corps en train de se construire et de se déconstruire dans l'interaction n'exclut pas un focus sur les ressources langagières. Ces dernières décennies, un débat s'est installé entre deux courants du féminisme autour de la matière et du discours, à savoir, entre la version butlerienne des études queer au début des années 90 et le «néo-matérialisme » incarné par des chercheuses telles que Rosi Braidotti $(1991,2014)$ et Karen Barad (2003), entre 
autres, prenant de la distance à la fois avec le mouvement postmoderne incarné par la figure emblématique de Butler et avec la tradition matérialiste classique marxiste (Dophijn \& van der Tuin 2012). Ces dernières reprochent à Butler, en faisant référence à son ouvrage Bodies that matter (1993), et à tout le féminisme d'inspiration postmoderne, d'avoir négligé le corps et sa matérialité au profit du discours dans les processus de construction du réel et $a$ fortiori du genre. De ce fait, selon ces autrices, si le féminisme postmoderne a réussi à interroger beaucoup de dichotomies telles que masculin/ féminin, nature/culture, hétérosexualité/homosexualité, il n'a pas réussi à questionner celle de "matière/discours » qui reste, selon ces chercheuses, encore intacte. Le projet de ce courant est ambitieux : se focaliser sur la matière et repenser l'articulation de celle-ci avec le discours (Alaimo \& Hekman 2008a : 6). Si ce nouveau type de féminisme, s'inspirant d'une part des travaux de Deleuze, Spinoza et Foucault, et d'autre part des travaux de l'écoféminisme et des recherches sur la science et la technologie (Science and Technology Studies), contribue d'une façon remarquable à mettre au centre de ses investigations la matière dans toute sa complexité, corporelle, spatiale, organique, il échoue là où il prétend dépasser le féminisme postmoderne. En effet, si selon les néomatérialistes le féminisme postmoderne n'arrive pas à rendre compte de la matière, au profit d'un idéalisme linguistique où "tout est discours", le féminisme néomatérialiste, à son tour, n'arrive pas à analyser les pratiques discursives des acteurs et des actrices sociales et se contente d'un vague renvoi au discours et à son intégration dans un cadre où la matière interagit avec le discours. De ce fait, la dichotomie matière/discours dont semble pétri le féminisme postmoderne n'est pas non plus résolue, me semble-t-il, par le féminisme néo-matérialiste, faute d'un positionnement théorique sur le discours et son intégration au sein d'un cadre holistique où discours et matière interagissent.

2 En partant du présupposé que le langage est appréhendé dans sa dimension relationnelle, incarnée, matérielle et temporelle et qu'il existe une matérialité linguistique qui se donne à voir et se construit dans la richesse et la complexité de répertoires multimodaux mobilisés par les participante-s pour accomplir des actions et performer le genre, la dichotomie corps/matière vs discours constitue une impasse théorique et épistémologique importante. Si ce problème est en partie résolu grâce à des contributions importantes au sein des approches praxéologiques du langage en questionnant la dichotomie entre corps et langage (C. Goodwin 2000, Mondada 2014, Bucholtz \& Hall 2016), cette question reste à mon avis encore ouverte dans les études de genre, queer et féministes. Si Braidotti, Barad ou Alaimo \& Heikman voient dans la matière et le corps un nouvel enjeu pour la théorie féministe s'opposant au linguistic turn, il faut rappeler que le langage est déjà matière et que la matière, qu'elle soit corporelle, spatiale ou artefactuelle, produit du sens en interaction. Cette vision est possible à condition d'appréhender le langage dans sa dimension irréductiblement holistique et multimodale (Mondada 2016) dans laquelle la grammaire, le lexique, la prosodie, les gestes, les regards, les postures, l'espace, les mouvements, la manipulation d'artéfacts, le toucher, la locomotion construisent ensemble l'intelligibilité de l'action, sans que l'analyse postule un ordre de priorité des unes, par exemple, le verbal, sur les autres. Dans ce cadre, il s'agit de changer d'optique et de se situer au sein de cadres analytiques dépassant les unités d'analyse linguistiques, comme par exemple le système d'activité située (Goffman 1961) ou les gestalts multimodales complexes (Mondada 2014) rendus pertinents par les acteurs et les actrices sociales en interaction et constitués par des assemblages mouvants de ressources sémiotiques parmi lesquels le 
corps, en interaction avec la parole, la prosodie, les objets et l'espace donne sens à l'action tout en étant configuré par elle. Comme le précisent Deleuze et Guattari (1980 : 13) « les agencements collectifs d'énonciation fonctionnent en effet directement dans les agencements machiniques, et l'on ne peut pas établir de coupure radicale entre les régimes de signes et leurs objets $»^{1}$. Ces nouvelles unités d'analyse doivent aussi rendre compte de la façon dont ces assemblages multimodaux renvoient à, et sont configurés par, une pluralité de contextes dans lesquels les normes de genre, les idéologies, la culture dans laquelle les acteurs et les actrices sociales interagissent ont un impact sur elleux, en remodelant leurs actions et en traversant leurs existences. Ce cadre d'analyse permet une double prise de distance à la fois par rapport aux critiques néomatérialistes à l'encontre du féminisme postmoderne et par rapport à Butler (1993) chez laquelle le corps est davantage un thème, tel qu'il est traité par les philosophes, les psychanalystes et les féministes, qu'une ressource en soi pour l'action. Il s'agit alors de considérer le corps moins en tant qu'objet de discours, un corps parlé, qu'en tant que corps parlant, en action et en interaction avec d'autres types de matérialités assemblées les unes dans les autres dans le cours des interactions. À partir de cette "troisième voie », entre Butler (1993) et le féminisme néo-matérialiste, un positionnement théorique est possible à condition d'interroger les travaux féministes sur la matière dans un cadre interactionnel, multimodal et anthropologique. Pour ce faire, je m'inspirerai des travaux qui, au sein des recherches sur le genre, le langage et la sexualité, se focalisent sur la dimension matérielle, corporelle et spatiale du genre dans une visée multimodale (M.H. Goodwin 2006, Machin et alii. 2016) et de ce qui, dans les études néo-matérialistes, va sous le nom d'une nouvelle "ontologie interactionniste " (Alaimo 2010 : 70) inspirée par les travaux de Whitehead:

Interactionism acknowledges both the agency of materiality and the porosity of entities. What I have termed "interactionism" has been inspired by a Whiteheadean process metaphysic [...] where the basic units of existence are phenomena (rather than physical objects) that are emergent from interactions. (Tuana $2008: 191$ )

C'est à l'intersection de ces deux courants que mes analyses se situent et qu'elles entendent ouvrir un espace de dialogue en traitant l'ontologie interactionniste des féministes néo-matérialistes comme un véritable objet d'analyse dans laquelle les matérialités corporelles, ici tactiles, sont irréductiblement imbriquées dans une multiplicité de contextes linguistique, corporel et extra-interactionnel.

Dans cet article, je présente d'abord les travaux qui se sont intéressés au toucher en tant que modalité sensorielle, interactionnelle et esthétique; ensuite, après avoir présenté les données constituant le matériau empirique de l'article, j'aborde l'analyse des pratiques tactiles en m'intéressant à ce que j'appelle le dédoublement tactile corporel dans l'interaction et une poétique et une politique de l'expérience tactile. Enfin, après un bilan clôturant la section analytique, je présente quelques conclusions en forme de bilan théorique et analytique pour une vision incarnée, sensorielle et créative du genre.

\section{Penser ensemble le genre et le toucher}

5 Le toucher est une technique corporelle, une ressource sémiotique mobilisée par les participante's pour percevoir et répondre au monde physique, pour accomplir l'intersubjectivité, construire et maintenir l'intimité, et de façon plus générale, pour faire l'expérience du monde, comme il en a été attesté par des travaux en psychologie 
(Gibson 1962) et en philosophie (Merleau-Ponty 1964, Deleuze 1981, Levinas 1961, inter alia) et en études culturelles (Paterson \& Dodge 2012). Le toucher permet de mettre au premier plan le rôle de la perception et des affects dans une vision de la connaissance qui est irréductiblement située, de questionner la neutralité du sujet percevant et la primauté de la vision dans la perception (Vasseleu 2015) et de penser à la connaissance en termes féministes : relationnels, empathiques et éthiques :

Thinking with touch can thus emphasize an ethical awareness regarding material consequences. This concerns knowing practices that take the chance to add relation to a world by involvement in touching and being touched by what we "observe". I seek a conception of touch that doesn't evoke a hold on reality with improved grasp, but rather engagement with the proximity of touching vision with slowness and care, attention to detail, definitively not hurried efficacy and cleanliness. (Puig de la Bellacasa 2009 : 310)

6 Le toucher est au centre également des pratiques somatiques et thérapeutiques. Il occupe une place importante dans les interactions entre sage femme et femme enceinte lors de l'accouchement (Boston Women's Health Book Collective 1973) et il ne peut, par ailleurs, être pensé en dehors des questions de consentement notamment en contexte de santé sexuelle et de violence obstétricale (Choulet 2019). C'est notamment grâce aux travaux de Luce Irigaray (1997) que le toucher, sous la forme de la caresse, qu'elle qualifie aussi de "geste-parole», devient ce qui permet de construire un lien social, intime, avec l'autre et de faire écho, grâce à ce contact entre les deux corps, à la dynamique interactionnelle entre un «Je» et un " $\mathrm{Tu}$ ». Les relations tactiles sont traversées aussi par des relations de pouvoir. Ainsi, comme l'a montré Erving Goffman (1956), dans les interactions à l'hôpital, le toucher joue un rôle important dans les interactions entre médecins et infirmières en rendant compte d'un rapport de force entre les participante's. Dans un travail sur les publicités dans les magazines, Goffman (1976 : 29) propose la notion de «toucher ritualisé » (ritualistic touch), ou de «toucher féminin " (feminine touch) pour désigner des configurations tactiles dans lesquelles est construite une image médiatique de la femme en tant que sujet soumis et fragile. Ainsi, on voit une main de femme toucher délicatement un parfum, ou bien un verre tenu par la main ferme d'un homme, ou encore une femme poser sa main sur la poitrine d'un homme à la recherche de réconfort. Si les hommes sont présentés comme des êtres dont la corporéité est stable, fixe et solide, celle des femmes apparaît irréductiblement fragile et vulnérable.

7 Grâce à une étude ethnographique fondée sur l'observation dans les espaces publics, Nancy Henley (1973) s'est penchée sur les droits au toucher (entendu comme un geste intentionnel de la main) dans les interactions dyadiques entre hommes et femmes, dans les espaces publics, en plein air. Selon cette chercheuse, le toucher est une des pratiques au travers desquelles plusieurs formes d'asymétrie sociale se donnent à voir et s'exercent: les hommes initient le toucher sur les femmes d'une façon plus importante que l'inverse en raison de la place occupée par les hommes dans notre société. Goffman dans son texte The arrangement between the sexes de 1977 arrive à peu près aux mêmes conclusions. Dans des interactions dans lesquelles les personnes ne sont pas intimes, les hommes touchent les femmes beaucoup plus facilement que l'inverse. Les hommes ont un "droit» au toucher sur les femmes et leurs pratiques tactiles sont vues alors comme un signe de protection, une pratique affective ou encore comme teintées d'ironie. L'étude pionnière de Henley a été par la suite largement commentée et retravaillée, et plusieurs biais méthodologiques y ont été mis en 
évidence : la nature intentionnelle du geste tactile, la non prise en compte d'autres variables comme la race, la classe, l'âge dans les pratiques tactiles, le type de situation observée, le type de toucher et sa fréquence, les parties du corps mobilisées dans le toucher, et la réaction de la personne touchée (Stier \& Hall 1984). De plus, la corrélation entre statut socio-économique et toucher, censée expliquer les différences et les asymétries de genre dans les relations tactiles, pose problème. Le statut socioéconomique est inféré par l'observation anonyme, il n'est pas considéré comme interagissant avec d'autres variables comme, par exemple, l'âge, et demande à être testé dans des contextes plus précis (Hall \& Veccia 1992). Dans le domaine de l'analyse des interactions (anthropologie linguistique et analyse de conversations), les travaux sur genre et toucher sont à ma connaissance pratiquement absents. Si les recherches conduites par Marjorie Harness Goodwin et Asta Cekaite (2018) sur le toucher dans les interactions en famille apportent une contribution fondamentale à l'étude des pratiques tactiles dans l'interaction, en s'intéressant davantage à la construction de la relation parentale qu'à sa dimension genrée, le genre y reste non analysé. De plus, que ce soit dans le domaine de la psychologie sociale, dans celui de l'analyse conversationnelle ou même dans les analyses d'Irigaray, la différence entre les sexes est donnée soit comme un point de départ soit comme l'horizon visé par les enquêtes. C'est dans les études culturelles que les catégories de genre sont véritablement questionnées. Le travail de Manning (2007) est exemplaire en ce qu'il se concentre notamment sur la manière dont le toucher dans le tango permet aux corps de se transformer sans cesse. Cette dynamique des corps rend les positions genrées - l'homme qui porte, la femme qui se laisse porter - beaucoup plus instables. On préfère alors parler, dans ce cas, de "leader » et de "follower", de rôles interchangeables, bien que teintés par des relations de pouvoir, dans un processus au sein duquel le genre est le produit (cf. engendering, ibid. : chapitre 4) plus que la cause des rapports sociaux. Contrairement aux travaux précédents, le genre cesse ici d'être un « déjà là » pour devenir, par le biais du toucher, un objet irréductiblement plastique et dynamique. Cette dynamique profondément transformatrice opérée par le toucher est abordée également par la théoricienne néomatérialiste Karen Barad (2012). En partant d'une perspective issue de la physique, Barad pense au toucher en tant qu'interaction électromagnétique, et, plus spécifiquement, à un processus par et dans lequel une infinité d'altérités - êtres, espaces, temporalités - sont suscitées et créées. C'est justement autour de ces altérités que je vais me pencher dans ce papier en montrant le caractère temporel, multimodal, interactionnel et profondément queer de ce processus en ce qu'il permet de notamment d'excéder et d'interroger la dyade soi-autre dans la relation humaine, et la dyade matière-discours dans l'interaction.

\section{Les données}

Les données constituant le matériau empirique de cet article sont issues de trois projets différents dont la mise en relation a trouvé sa raison d'être grâce à l'émergence et à l'évidence de l'objet "pratiques tactiles en milieu féministe et LGBTQ " dans ma trajectoire scientifique. Le premier est une enquête de terrain menée dans un atelier drag king à Bruxelles dans laquelle je me suis intéressé à la construction multimodale des masculinités chez les drag kings (Greco 2018). Le deuxième concerne une enquête que j'ai menée en Italie (en Lombardie et en Toscane) auprès de cinq femmes ayant participé dans les années 70 aux groupes de conscience féministe. Le troisième porte 
sur l'émergence et la circulation du concept de performance, et des pratiques performatives, dans les arts et les sciences sociales des années 50 aux années 90 aux États-Unis et en Europe. Ce troisième projet a transformé mes pratiques spectatorielles en activité ethnographique faisant des performances que je regardais en tant que spectateur un matériau pour l'observation et l'analyse. Le focus sur le toucher en tant que dispositif de construction et de déconstruction du genre a permis une mise en relation inédite de ces trois projets/corpora. Cette articulation est rendue possible par trois points qu'il convient ici de distinguer par souci de clarté même s'ils peuvent être pensés comme s'articulant entre eux. D'abord, l'objet du toucher. Il permet en effet de considérer, après coup, que des projets et des terrains qui en principe n'étaient pas pensés comme allant ensemble donnent lieu à de nouvelles perspectives théoriques et à de nouveaux agencements analytiques. Ensuite, la prise en compte de l'art contemporain dans mes recherches. Elle permet de considérer les performances artistiques comme des objets interactionnels, des sources d'inspiration théorique (Greco 2016, 2017) et de penser l'articulation entre l'analyse des pratiques artistiques et celle des pratiques langagières comme produisant des éclairages nouveaux et inattendus. Dans ce cadre, il s'agit de créer, grâce à un focus sur l'objet, des assemblages inédits et surprenants entre drag kings, féministes des années 70 et art contemporain. Cette mise en relation est proche d'une expérience artistique. Elle permet, en effet, de créer, à partir du même objet, le toucher, et du cadre politique sous-jacent aux ateliers drag king, aux groupes de conscience et à la performance féministes, des assemblages inusuels et de donner corps à de véritables opérations de traduction culturelle (Bhabha 1997) en ce qu'elles favorisent l'émergence d'un espace de rencontre entre plusieurs types de discours, de registres, de cultures. En m'inspirant des travaux des plasticien'ne's dans lesquels le discours, la matière, l'espace et les corps interagissent en même temps et au même niveau en donnant lieu à des images et à des objets esthétiques inédits et qui font sens, la mise en relation entre différents corpora relève pour moi du même processus, elle fait écho à l'approche holistique et multimodale convoquée sur le plan théorique et à la notion d'assemblage que je mobilise à plusieurs reprises pour rendre compte d'objets composites et mouvants tels que les corps et le genre dans une phase de devenir incessante :

What is an assemblage? It is a multiplicity which is made up of many heterogeneous terms and which establishes liaisons, relations between them [...]. Thus, the assemblage's only unity is that of a co-functioning: it is a symbiosis, a "sympathy". It is never filiations which are important, but alliances, alloys; these are not successions, lines of descent, but contagions, epidemics, the wind. (De Landa 2016 : 1)

\section{Les analyses}

9 L'analyse des corpora présentés dans cet article permet de rendre compte de la dimension tactile et interactionnelle dans les procédés de construction et de déconstruction du genre et de focaliser notre attention sur la façon dont le toucher permet de produire et de projeter un·e tiers acteur/tierce actrice imaginéee et idéalisée.e. De ce fait, le genre est étudié au prisme non seulement de l'interaction et de la multimodalité mais aussi de l'imagination et de l'expérience. Ce focus sur l'imagination et l'expérience permet de rendre compte de la dimension esthétique et politique des pratiques tactiles en ce qu'elles permettent d'avoir un accès aux possibles engendrés 
par le toucher en interaction dans le dépassement de la relation dyadique dans l'interaction et dans le projet d'un corps malléable et plastique.

\section{Imaginer un nouveau corps ensemble : un cas de dédoublement tactile corporel}

Dans cette section, je vais me pencher sur l'activité de maquillage telle qu'elle se déroule dans un atelier drag king. Cette activité est structurée en trois temps. D'abord, une première phase dans laquelle la/le novice demande à l'experte d'intervenir (directement ou indirectement) dans la pratique de maquillage/transformation du corps. L'experte peut aussi s'auto-sélectionner pour aider la/le novice à comprendre comment opérer la transformation. Ensuite, une deuxième phase dans laquelle on assiste à un moment de négociation et d'explication sur les modalités de maquillage et de transformation. Dans ce cadre, l'experte a recours à son propre visage pour rendre intelligibles les modalités de fabrication corporelles. Enfin, la troisième phase, qui coïncide avec le début du maquillage proprement dit, dans laquelle l'expert·e maquille la/le novice, et suite à laquelle ille pourra terminer le maquillage toutee seul.e. Je vais me concentrer sur la deuxième phase, en laissant de côté le maquillage proprement dit dont l'analyse ne permet pas de rendre visible le phénomène qui m'intéresse ici. L'extrait sur lequel je vais m'attarder ${ }^{2}$ commence avec une question de Camille sur les modalités de construction d'une barbe (ligne 1$)^{3}$.

Image 1

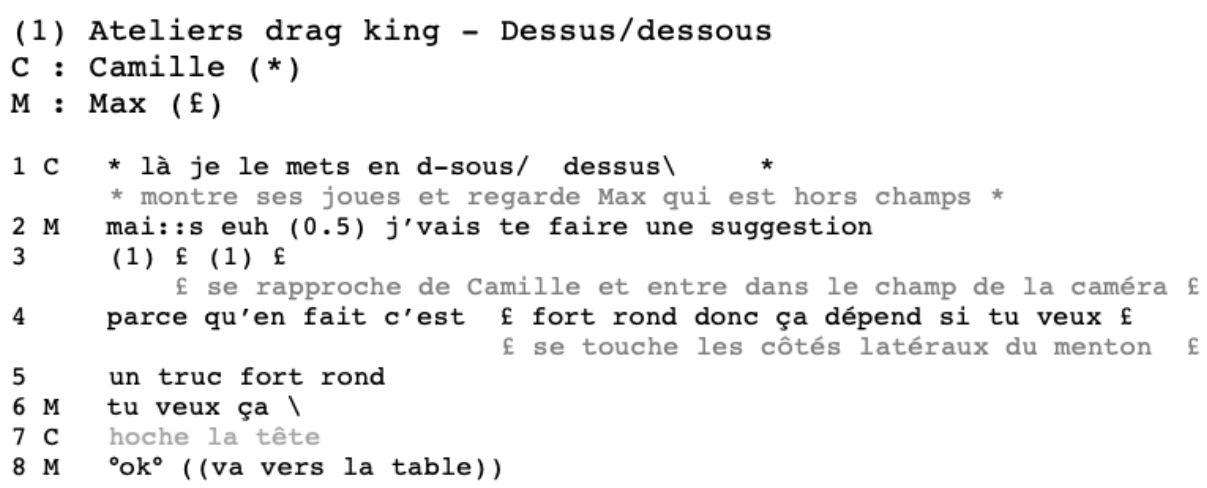

11 La question de Camille (1. 1) est interprétée par Max (1.2) comme une demande d'aide, comme le montrent d'abord son déplacement dans l'espace vers le corps de Camille (1.3), véritable objet de discussion et de travail, et ensuite sa prise de parole (1. 2-6), qui ouvre une séquence insérée au sein de laquelle Max va faire quelques suggestions et poser quelques questions à propos de la forme désirée par Camille. Cette séquence donne lieu finalement à une autre suggestion de travail (1. 9-12) : 
Image 2

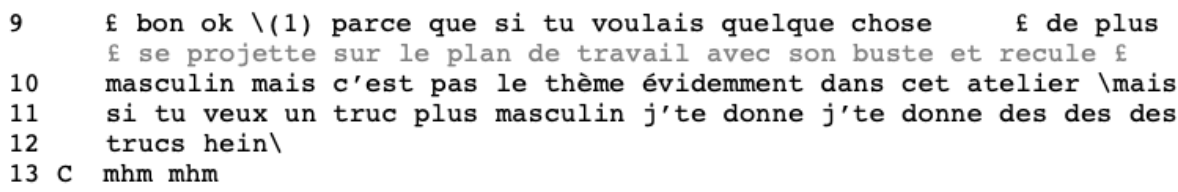

12 Cela est rendu intelligible aussi par le double mouvement corporel de Max dans l'espace, (1. 9 non verbal), d'abord en se projetant vers la table de travail et ensuite en reculant pour formuler une autre suggestion qui sera acceptée par Camille à la ligne 13.

13 L'acceptation de Camille va donner lieu à une série d'instructions (1. 14, 16, 19, 23, 26, $30,31)$ pour la construction d'un personnage «plus masculin » (1. 11) comme dans la suite :

Image 3

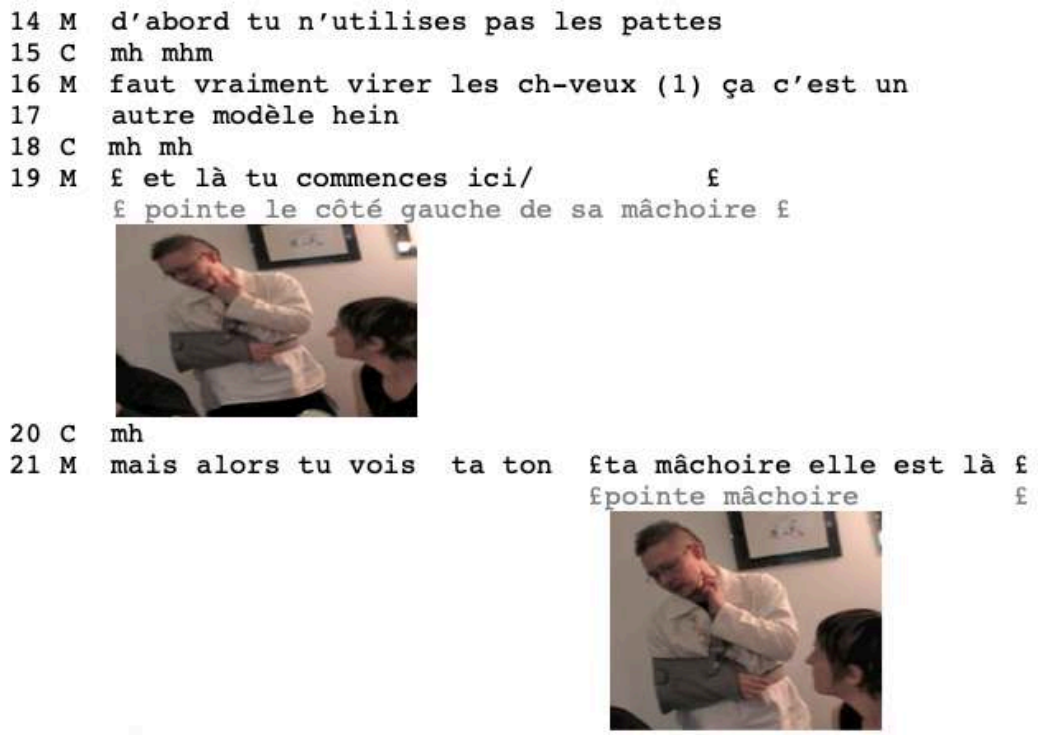


Image 4

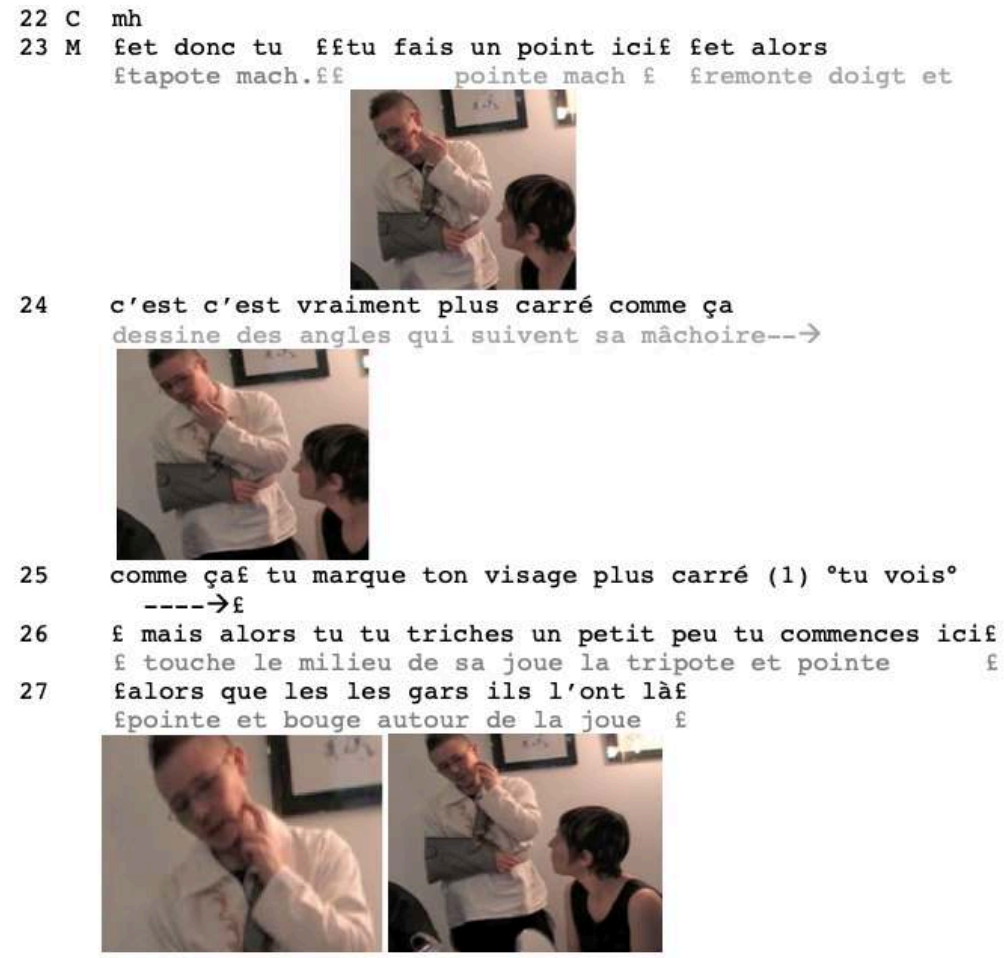

Image 5

28 fmais leur mâchoire tu sais pas

Eredessine une mâchoire

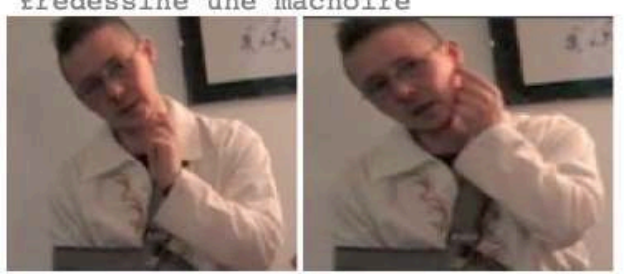

29 tu sais pas la redessinerf en vrai quoi

en dehors de sa mach. $--\rightarrow £$

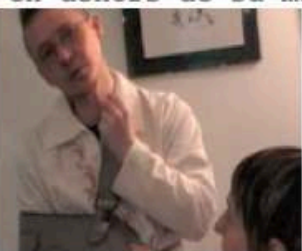

$30 \mathrm{C} \mathrm{mh} \mathrm{mh}$

31 M fdonc tu dois la la fausser sur ton visagef

ffait un angle en touchant la machoiref

32 c'est pour ça que tu tu mets tu la mets un peu plus hautf tu vois

Les gestes que Max produit sur son propre visage, d'abord au niveau des cheveux puis au niveau de la mâchoire, produisent un travail très important sur son corps. Ces pratiques tactiles opèrent un désancrage du visage de Max de lui-même, ce que j'appelle un « dédoublement tactile » du corps, ici la partie du corps «mâchoire ». C'est un procédé par lequel le visage de Max, cesse, par le toucher, d'être son visage pour devenir un visage à partir duquel le travail de transformation peut être effectué. Tout 
en restant son visage, c'est sur celui-ci que cette démonstration se fait, ce visage devient autre, se dédouble. Il devient, de la sorte, un visage " modèle », un support, qui se donne à voir à Camille et qui pourrait être le sien une fois qu'il sera mis au travail. C'est un visage dans la mesure où il se donne à voir comme une entité descriptible, observable, évaluable (accountable cf. Garfinkel 1967), projetable dans le futur une fois que Camille se mettra au travail.

Ce travail d'imagination collective est rendu possible par des procédés qui sont à la fois de décontextualisation de la mâchoire - elle cesse d'être la mâchoire de Max grâce au toucher - et de recontextualisation, en devenant la mâchoire de Camille, « ta mâchoire elle est là » (1. 21) et celle des gars, « les gars ils l'ont là ", « leur mâchoire » (1. 27-28).

Les pratiques tactiles de Max sur son propre visage permettent donc d'accomplir une apparition ontologiquement impensable : Camille peut voir sa propre mâchoire et celle des gars sur le visage de Max touché par lui-même.

Ce travail de transformation, d'imagination et de dédoublement tactile se poursuit lorsque Max donne des indications telles que «tu dois la la fausser sur ton visage » (1. 31) ou «tu la mets un peu plus haut » (1.32), donnant corps et mettant en scène la mâchoire de Camille comme un objet de travail plastique. Si, pour Deleuze (1981: 146), on peut parler de "vision haptique " pour une œuvre d'art où il est possible d'identifier et d'appréhender les mouvements de la main qui ont dessiné une forme, ici le corps se donne à voir en train de se faire, observable grâce à la conjonction d'une multiplicité de ressources tactiles, visuelles, posturales, grammaticales.

Dans le prochain extrait, Max poursuit le travail d'imagination d'une barbe possible avec Camille :

Image 6

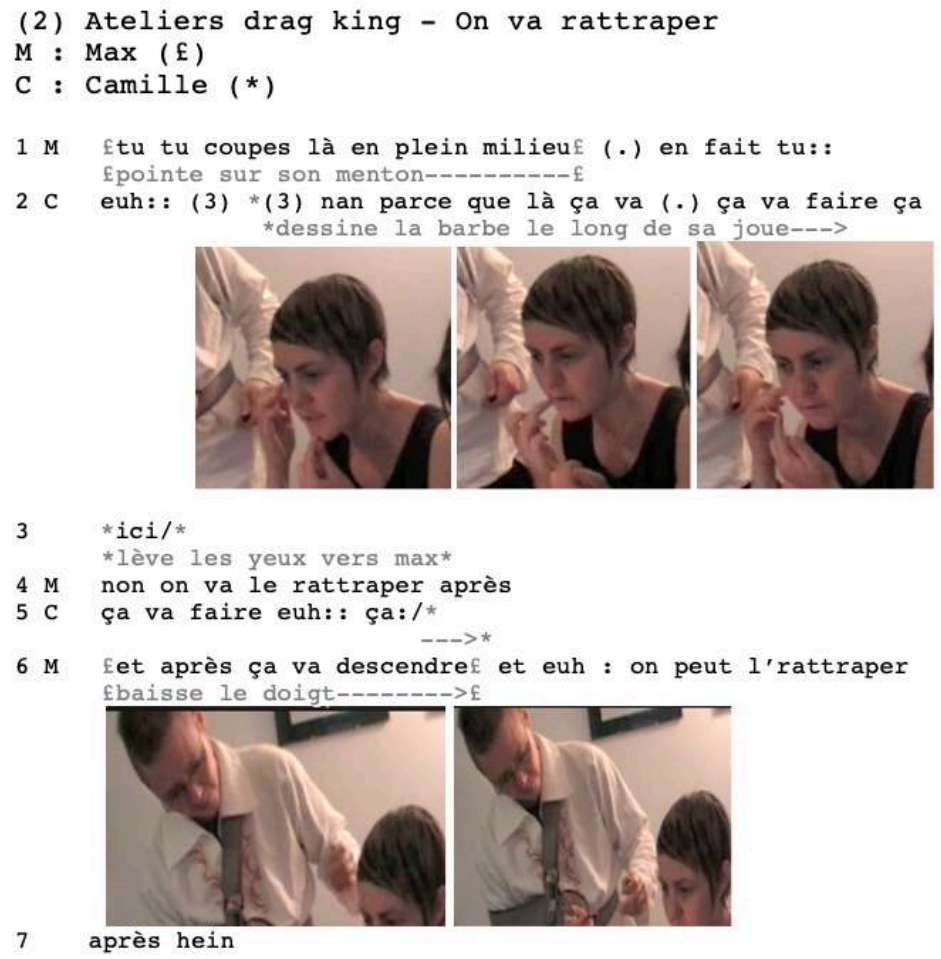


L'extrait commence avec une instruction formulée par Max sur le mode impératif (1. 1) tout en pointant sur son menton pour indiquer et projeter la partie du corps investie par la transformation à venir. Cette première partie de la paire (instruction/action réalisée ou non réalisée) déclenche une réaction de la part de Camille sur un mode non préféré remettant en question l'instruction de Max comme le montre le formatage initial du tour de Camille (1.2, marque d'hésitation, pause, particule négative, account " parce que... »). C'est à partir notamment de l'account de Camille (préfacé par « parce que » 1.2) que prend place un travail imaginatif sur la transformation genrée de son visage. Il se nourrit d'une multitude de ressources sémiotiques imbriquées : le toucher ayant la fonction de cartographier une zone pertinente pour la transformation (la joue sur la partie droite du visage) et de rendre publique une transformation à venir, la vision sur le miroir, et une construction grammaticale dont le format se stabilise au fil de son tour. D'abord, "ça va» (l.2), ensuite, après une micro-pause (.), une reformulation du premier «ça va » en « ça va faire ça » (1. 2), et enfin le déictique « ici » (1. 3). L'imbrication entre la marque spatiale « ici », avec l'intonation montante (« / ) et le regard vers Max rend le projet de transformation corporelle genrée évaluable et soumis à révision. La réponse négative et la proposition de Max (1.4) entraine chez Camille une autre proposition avec une intonation montante (1.5). Dans cette proposition, la forme de la barbe n'arrête pas de se construire. Elle s'accomplit par et dans un véritable travail d'imagination collaborative (Murphy 2004) poursuivi par Max à la ligne 6 comme l'indique le connecteur "et", en position initiale de son tour, indexant un lien de cohésion syntaxique avec les propositions de Camille. Le travail de transformation genrée ici à l'œuvre est collectif, accomplie par la dyade expert/novice endossée par Max et Camille, multimodale, prenant forme grâce à l'imbrication entre la syntaxe, le toucher et la vision (Nishizaka 2003), donnant lieu à un procédé de dédoublement tactile corporel. Dans ce cadre, comme nous l'avons vu précédemment, le toucher permet de transformer une partie du corps, ici la joue, en une partie du corps possible, plastique, en train de construire. Que ce soit chez Max ou chez Camille, le dédoublement tactile corporel permet de poser un tiers dans l'interaction, un corps idéalisé, imaginé, réifié par et dans l'assemblage entre plusieurs matériaux sémiotiques.

\section{Pour une poétique et une politique de l'expérience}

Dans cette section, nous allons poursuivre ce travail de production d'un tiers imaginé, futur, possible, dans un autre contexte mais néanmoins lié au précédent à la fois par le partage d'une même technique corporelle, le toucher, et d'un même horizon politique, le féminisme et l'activisme LGBTQ. Que ce soit dans les années $70 \mathrm{chez}$ les militant·e's féministes et de libération du mouvement homosexuel, ou bien chez les drag kings, aujourd'hui, nous avons ce que l'on pourrait appeler un socle commun partagé par ces deux communautés: le corps en tant que lieu privilégié de l'expérience politique et poétique, voire existentielle. L'expérience par et dans le corps constitue sûrement l'un des accès privilégiés à la connaissance du monde. La tradition philosophique issue du pragmatisme considère l'expérience dans les termes d'une interaction de l'organisme avec son environnement aboutissant à une transformation en participation et en communication (Dewey 1934 : 60). Contrairement à toute une tradition philosophique remontant à Aristote et à Kant selon laquelle l'art se distingue de la vie et de l'éthique, l'expérience esthétique telle qu'elle est conçue par le pragmatisme met à mal la dichotomie entre art et vie, corps et esprit, pour rejoindre d'une part l'esthétique de 
l'existence de Michel Foucault (1984) visant à faire de sa propre vie une œuvre d'art, et d'autre part, à considérer les interactions de la vie quotidienne comme un matériau esthétique (Kaprow 1993). Que ce soit dans les ateliers drag king, dans les pratiques de conscience des féministes italiennes des années 70 , ou encore dans les performances artistiques contemporaines, nous assistons à un remodelage du corps et des existences des actrices et des acteurs, qui excède l'enveloppe corporelle des sujets et les interactions dans lesquelles illes sont prise's pour viser d'autres corps et d'autres relations possibles. C'est dans ce sens que la reformulation de ces pratiques au prisme d'une poétique et d'une politique de l'expérience nous semble particulièrement adéquate ${ }^{4}$. Le terme de "politique de l'expérience » est utilisé par Corrado Levi (2010), activiste gay italien, pour désigner les pratiques militantes d'un mouvement révolutionnaire pour la libération des personnes homosexuelles, le FUORI (Fronte, Unitario, Omosessuale, Rivoluzionario Italiano [Front Unitaire Homosexuel Révolutionnaire Italien]). Il est issu de la contre-culture des années 70 et il est lié aux pratiques de l'auto-conscience. Les pratiques de l'auto-conscience sont constitutives de la théorie et de la pratique du féminisme (McKinnon 1982). Elles permettent, dans un cadre collectif et à partir de sa propre expérience personnelle, une re-subjectivation de celles et de ceux qui la pratiquent. Elles donnent notamment la possibilité de prendre conscience des liens existants entre son expérience personnelle et les structures sociales (famille, école, travail, etc.) ainsi que la valeur politique de sa propre position au sein de l'échiquier social. Ce sont des pratiques analytiques en ce qu'elles obligent les sujets à analyser leur propre comportement et celui des autres, mais aussi politiques et subversives car elles permettent une prise de conscience et une volonté de changer les structures de domination de la société. Dans un texte traduit et republié par la revue Genre, Sexualité et Société, voici comment Corrado Levi s'exprime à propos du toucher une fois sorti de ces réunions dans lesquelles on pratiquait l'autocoscienza :

Personnellement, en sortant des premières réunions, je me suis rendu compte que je tenais souvent quelqu'un par l'épaule ou que nous marchions main dans la main, et, avec grand étonnement, je me suis aperçu que personne n'y faisait attention, chacun pensant seulement à lui-même. Les seuls qui réagissaient le faisaient, me semble-t-il, pour exprimer une sorte de bonheur ou pour manifester leur plaisir de voir quelqu'un assumer avec courage d'être comme il veut, indépendamment du fait qu'il soit ou non homo. (Levi 2010)

Dans le contexte des années 70, en Italie, le geste de prendre une personne de même sexe par la main, dans un espace public, est un acte politique et poétique : il impose de nouvelles subjectivités et de nouvelles affectivités, et il permet de faire de sa propre vie un laboratoire à la fois existentiel et social. Dans une enquête que j'ai menée avec des féministes italiennes ayant participé dans les années 1970 à des groupes de conscience où les femmes se réunissaient pour parler de leurs propres expériences et acquérir ainsi un vocabulaire commun et une conscience de soi, je me suis rendu compte à quel point le toucher pouvait jouer un rôle considérable dans la construction de nouvelles modalités relationnelles et interactionnelles comme dans l'exemple suivant: 


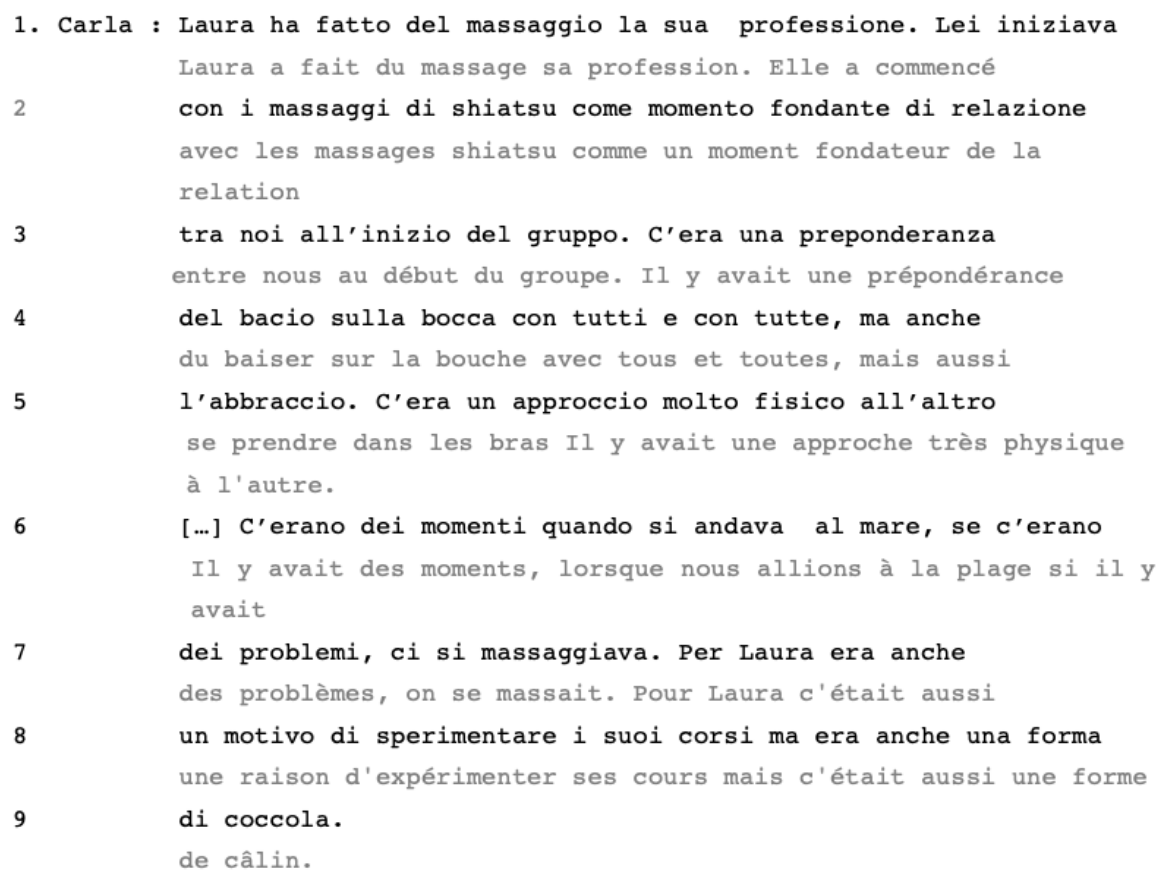

Carla, féministe et lesbienne, ancienne militante du Parti Radical, issue de la galaxie de la gauche libertaire et libérale italienne, est aujourd'hui professeure d'éducation physique dans les écoles. Le travail sur le corps a accompagné toute sa vie et a même donné un sens à son identité de lesbienne. Avant de travailler dans les écoles, elle a géré une salle de sport avec son ancienne compagne, en province, en Italie, qui était vite devenue une sorte de lieu repère pour la communauté lesbienne et gay habitant dans la région. Dans l'entretien, elle fait référence à plusieurs pratiques tactiles que les femmes dans les années 70 pratiquaient entre elles à la fois dans les groupes de conscience et à l'extérieur lorsqu'elles sortaient ensemble. Un véritable répertoire d'action tactile est ainsi mobilisé par les membres de ce groupe, notamment par Laura, masseuse shiatsu (l. 1), une amie de Carla. Ainsi, nous avons le massage, conçu comme un véritable outil de construction de nouvelles relationnalités et affectivités (1. 2, 8-9) dans le groupe (1. 2-3), mais aussi en tant qu'outil d'expérimentation professionnelle (1. 7). Le baiser sur la bouche (1. 3-4) et le fait de se prendre dans les bras (1. 4-5) sont appréhendés par Carla comme des ressources corporelles donnant lieu à une approche très physique à l'autre (1.5).

Cette nouvelle modalité relationnelle dans laquelle toucher, affects et parole sont assemblés est mise en avant aussi par Giovanna, résidente dans la même ville que Carla, aujourd'hui fonctionnaire à la mairie, ancienne militante du Parti Communiste Italien, et ayant fréquenté avec Carla les mêmes lieux de militance féministe : 
Image 8

(4) Autocoscienza Giovanna 1

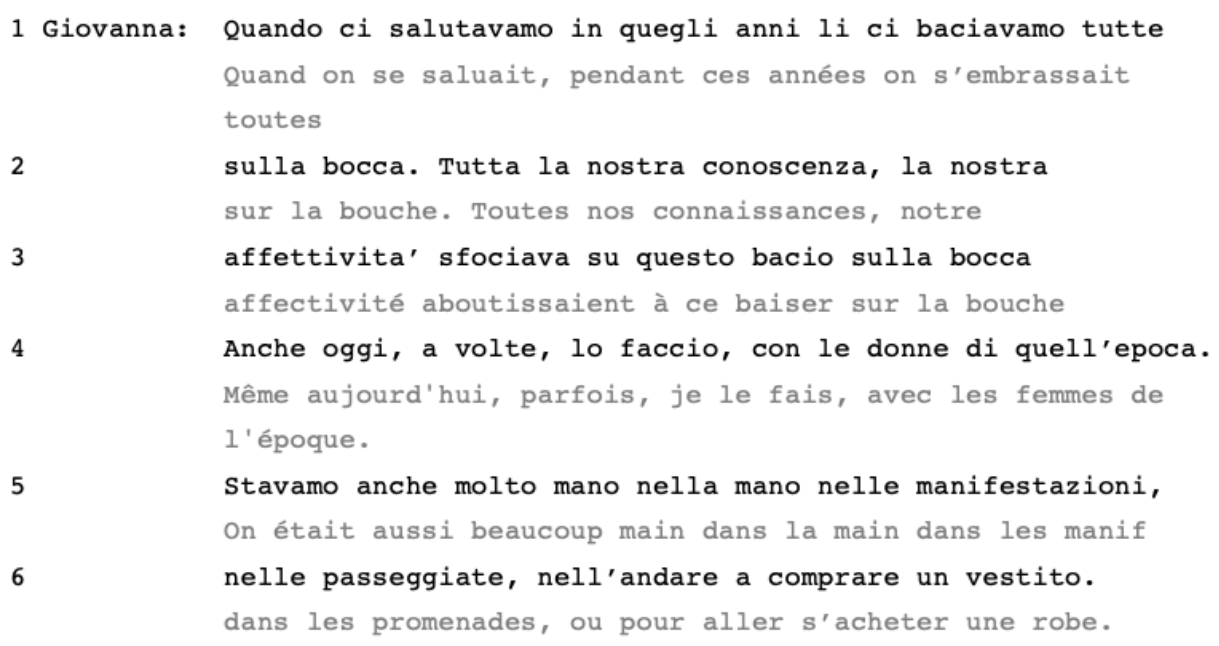

Chez Giovanna, le toucher se décline surtout avec la baiser sur la bouche et le fait de marcher main dans la main dans un espace public. Giovanna situe cette pratique corporelle dans une double temporalité : celle des années 70 (dont le référent est vague dans l'extrait mais partagé par Giovanna et moi-même au début de l'entretien) et celle d'aujourd'hui comme le montre le repère adverbial «oggi» (" aujourd'hui », 1.4) pour rendre compte d'une pratique qui se poursuit dans le temps avec les mêmes personnes qu'à l'époque. Pour Giovanna, ces pratiques permettent de nouvelles modalités relationnelles, une fusion des corps pour reprendre une expression de ZancariniFournel (2004: 210), et constituent un lieu d'observation et de concrétisation privilégiées du savoir et des affects vécus. Lea, militante féministe historique que j'ai rencontrée à Milan, autrice de nombreux essais et ayant contribué avec Carla Lonzi à installer la pratique de l'autocoscienza en Italie (Melandri 2002) me parle aussi du baiser sur la bouche et $\mathrm{du}$ fait de se prendre dans les bras comme de pratiques irréductiblement féministes : 
Image 9

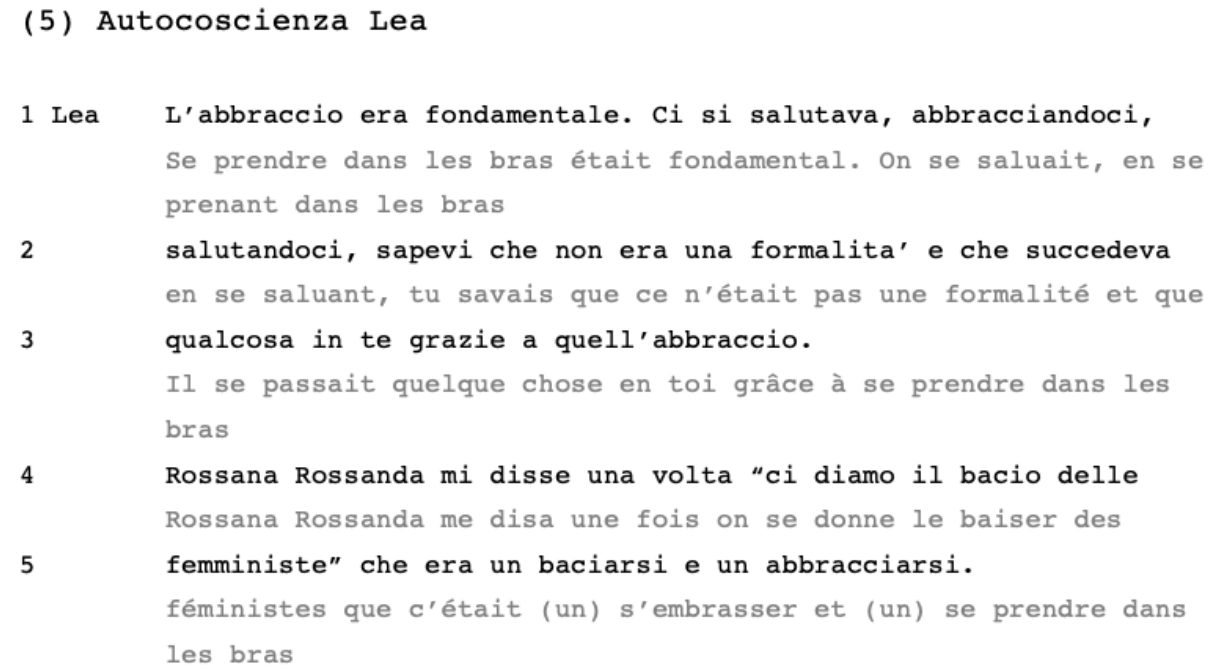

Ces pratiques tactiles sont pensées et vécues en dehors d'un cadre formel (1.2), ayant $\mathrm{du}$ sens et déclenchant un possible changement dans les existences des personnes (1. 2-3). Le baiser sur la bouche, par une technique de discours rapporté dans laquelle Lea fait référence à un témoignage de Rossana Rossanda, figure importante du communisme dissident italien, devient une pratique féministe (1. 4-5) reformulée par la suite dans son tour (1.4-5) comme « un s'embrasser et un se prendre dans les bras». Cette vision du savoir praxéologique, irréductiblement situé, est présentée dans le prochain entretien avec Giovanna :

Image 10

\section{(6) Auto-conscience Giovanna 2}

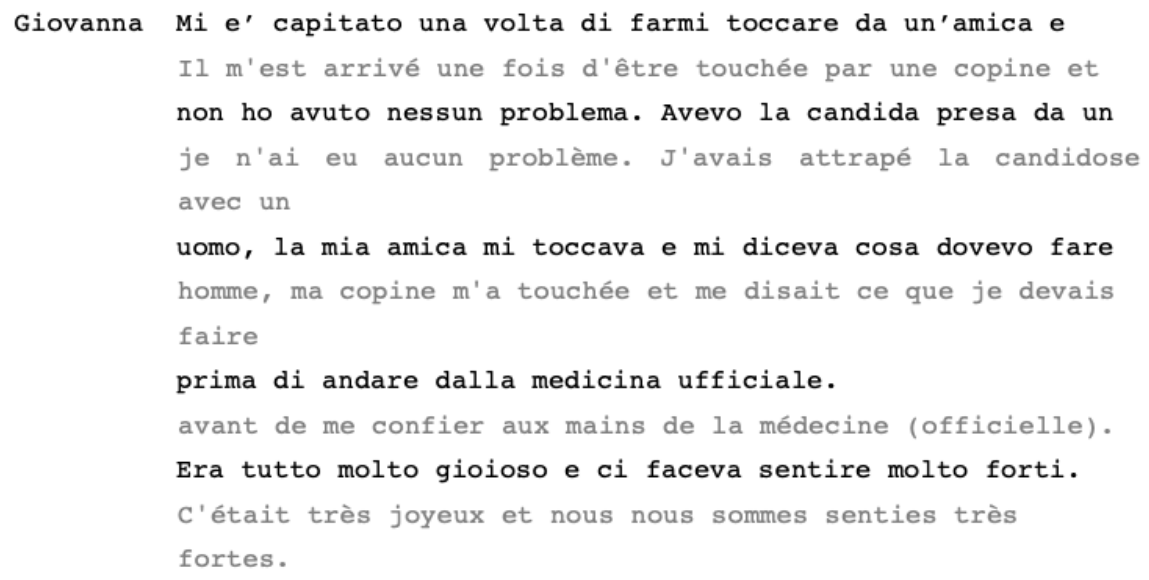

Le toucher, dans cet extrait tel qu'il est verbalisé par Giovanna, permet, dans un cadre joyeux et étranger à la relation de domination "médecin'e/patiente e ", de développer un savoir collectif et militant sur et par le corps et de construire un sens d'empowerment (1.5) pour les femmes. Dans ce cadre, le discours de Giovanna, surtout lorsqu'elle fait allusion à la "médecine officielle» renvoie à toute une littérature féministe dans 
laquelle les femmes, en luttant contre le pouvoir des médecins hommes sur leurs corps, ont décidé par un acte d'écriture collective de rédiger un texte pour les femmes à partir de leur expérience corporelle (Boston Women's Health Book Collective 1973, ZancariniFournel 2004).

\section{Ces corps qui touchent}

Pour reprendre et paraphraser le célèbre titre de l'ouvrage de Judith Butler, « Ces corps qui comptent » (Bodies that matter, 1993), où finalement le corps dont il est question est un corps entendu comme objet de discours par la philosophie et la psychanalyse, un corps parlé plus qu'un corps parlant ayant sa propre agentivité, nous avons affaire ici à des corps qui parlent, ou mieux, qui touchent, dans une pluralité de situations.

D'abord, dans les ateliers drag king. Ici, le toucher est considéré comme un dispositif de construction et de déconstruction d'un corps imaginé et désiré, une ressource par et dans laquelle le corps devient une surface partageable publiquement, remodelée sans cesse, à toutes fins pratiques, dans le cadre d'une scène dans laquelle les acteurs et les actrices sociales montrent et performent les modalités de construction corporelle en faisant de leur propre corps un support pour l'imagination et l'expérience.

Ensuite, dans l'enquête sur les groupes de conscience. Ici, nous avons vu comment le toucher pouvait jouer un rôle important dans la mise en place de nouvelles modalités relationnelles mais aussi en tant que pratique de connaissance de et sur son propre corps. Dans ces deux contextes, le toucher permet de renvoyer à une réalité ancrée dans un autre espace-temps que celle des interactions et des expériences vécues par les participante's à un moment $t$ de leur existence. Il permet, en effet, d'imaginer d'autres corps à venir ou situés à l'extérieur de l'espace et du temps de l'interaction, et de penser à de nouvelles façons d'être ensemble dans le monde qu'on expérimente par et dans le toucher.

Le toucher constitue également une source d'inspiration importante pour la cinéaste lesbienne nord-américaine Barbara Hammer. Dans une interview qu'elle donne dans le cadre de sa rétrospective au Jeu de Paume ${ }^{5}$, elle s'exprime ainsi : 
Image 11

(7) Interview Barbara Hammer Jeu de Paume

$\mathrm{BH}$ : Barbara Hammer

$1 \mathrm{BH}$ My sexuality did influence my influence then my early

film work and it comes down to a philosophy that I

developed. When I touch a woman whose body is similar to

my own I increase my sense of touch : all my hairs on my

body, all my skin, I'm more aware of my movement in the

world. This became crucial to my cinema because I want

to connect

sight

and touch.

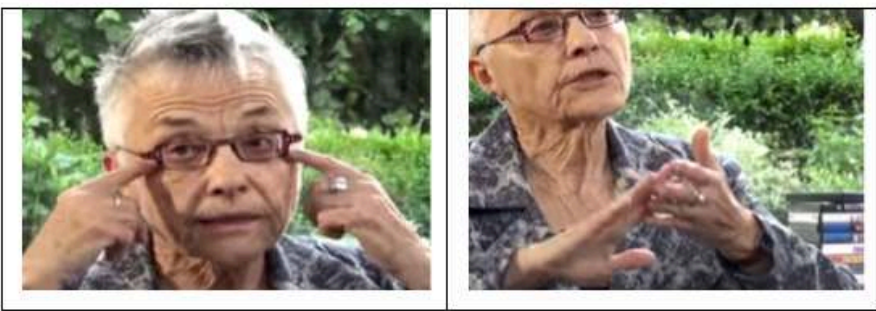

Image 12

8

The screen, the eyes

9

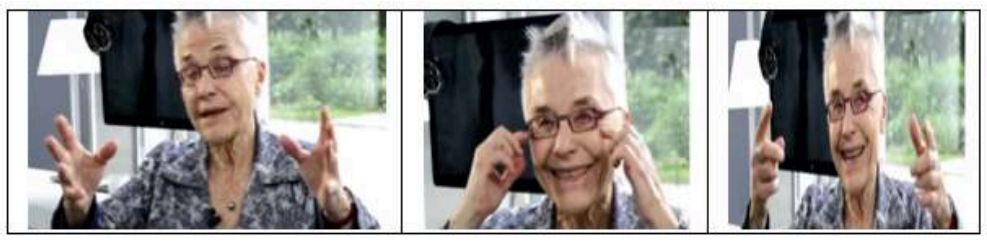

and the sense of the body for the audience not just for

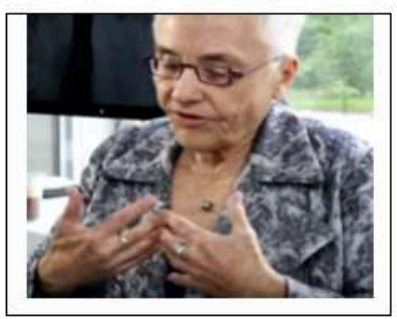

10 me

Dans cet entretien, Barbara Hammer rend compte d'un processus de création. Le cinéma qu'elle a développé au cours de ces longues années est issu d'une expérience corporelle précise et liée à sa sexualité. Le fait de toucher le corps d'une autre femme (1.3) rend sa conscience corporelle particulièrement accrue, au niveau de la perception tactile (1.4) mais aussi kinesthésique, en ayant un impact sur ses propres mouvements dans le monde (1.5-6). Ici, comme dans les exemples précédents, l'expérience tactile permet de sortir de l'instant $t$ de l'expérience pour se projeter dans d'autres espacetemps : celui de la création cinématographique et de la conception d'un cinéma dans lequel la vue est inséparable du toucher (1. 6-9). 

à d'autres types de relation entre public et performeuree's grâce à la mise en place d'une relationnalité tactile entre les deux sujets comme dans l'exemple de Goodnight, Peeping Tom (2016), une performance de l'artiste italienne Chiara Bersani dans laquelle les performeure's accompagnent un groupe de cinq spectateure's dans un véritable parcours haptique dans lequel la vision, le toucher et les assemblages corporels forment la chair d'une expérience d'une durée d'une heure. Les «spectateur·e• sont accompagnée's dans une pièce où l'on assiste à des scènes dans lesquelles les performeure's se regardent, se touchent, nous regardent, nous touchent. La disposition des corps dans l'espace est mobile, les performeure's bougent, sont assisees, forment des groupes et tiennent compte de nos présences dans l'espace dans une dynamique typiquement interactionnelle. De ce fait, les corps tiennent compte des mouvements des autres en donnant forme à des ajustements continus dans l'espace et à des formats en devenir instables. Les regards et les formes des assemblages corporels et interactionnels nous interpellent sur notre présence, genrée et sexualisée, et nous amènent à nous interroger sur la possibilité d'une action à venir. Les possibilités relationnelles offertes par le toucher peuvent constituer un des éléments centraux de l'être ensemble dans les milieux queer, non binaires, prônant une sexualité ou une sensualité non péno-centrée. Lors de mes participations à des événements organisés par des groupes queer, il m'est souvent arrivé d'assister à des situations dans lesquelles le toucher sous forme de massage ou de caresse était une pratique courante ou prévue dans le répertoire d'action du groupe. Lors d'une performance proposée par un groupe des Radical Fairies à Paris à l'espace Point Éphémère, l'une des animatrices du collectif avait proposé des massages au public. Ces massages ne constituaient pas une rupture par rapport à l'événement en place. Ainsi, tout en regardant le spectacle, il y avait toujours un couple dans lequel l'un massait et l'autre se faisait masser avec des huiles de massage. Ce toucher permettait de penser à d'autres façons d'être ensemble dans un espace public et safe et à d'autres modalités de percevoir un spectacle. De ce fait, on peut participer à une performance et se faire masser en même temps en effaçant ainsi la frontière entre vie et art, privé et public. Cette tactilité provoque à la fois un relâchement dans les postures, une invitation au massage et la proposition d'une intersubjectivité tactile. Le "calinodrome", une idée conçue par le groupe Lillois LGBTQ Les flamands roses, désigne des espaces safe dans lesquels un autre type de contact, de sensorialité entre les participante-s, est possible. Il se fait par le toucher et en dehors des relations sexuelles. C'est une option qui privilégie la sensualité à la sexualité et qui se situe politiquement dans une logique visant à entrer en contraste, par ces pratiques et par ces espaces, avec une idée de la sexualité consommée dans des lieux où les corps normés et valides sont les « maitres du monde ».

\section{Quelques conclusions : Faire monde avec le toucher}

L'analyse du toucher dans une pluralité de contextes (ateliers drag kings, pratiques artistiques et groupes de conscience) m'a permis de montrer comment le toucher peut être pensé comme une modalité de construction et de déconstruction du genre et des relationnalités ainsi que comme une porte d'entrée pour questionner et repenser les relations entre discours et matière. Concernant ce dernier point, j'ai montré comment une analyse multimodale rendant compte de l'imbrication entre la matérialité et la

GLAD!, 07 | 2019 
plasticité corporelle, d'une part, avec les ressources grammaticales et gestuelles, d'autre part, permet à la fois de sortir d'une perspective dichotomique selon laquelle une attention au discours exclurait de facto la matérialité des corps, et de prolonger le programme néo-matérialiste en donnant de la chair empirique et temporelle à la dimension interactionnelle invoquée par les théoriciennes se réclamant de ce champ de recherche.

34 J'ai aussi montré comment cette approche permet de penser au genre en termes d'assemblages mouvants, se formant et se décomposant au gré de l'action, par une multiplicité de ressources multimodales, affectives et sensorielles, en m'inspirant ainsi des travaux de Deleuze et Guattari (1980) et de De Landa (2016). Ces assemblages constituant et déconstruisant le genre au fil du temps de l'action ne sont pas uniquement mouvants et dynamiques. Ils sont aussi esthétiques en ce qu'ils mobilisent, au cours de leurs accomplissements, l'imagination et l'expérience, deux facteurs constitutifs de la performance en tant que genre artistique. Ils sont, enfin, performatifs. Ils ont en effet un impact transformateur sur la plasticité genrée des corps et des relations et permettent de penser à des corps en excès par le biais de la projection et de la présence d'un tiers imaginé et idéalisé. Que ce soit dans les ateliers drag king, dans les groupes de conscience ou dans les pratiques artistiques dont j'ai rendu compte, la matière corporelle et spatiale et le discours concourent, avec le toucher et les affects déployés par les participante·s, à la construction et la déconstruction du genre et à de nouvelles modalités relationnelles.

Les cas que nous avons examinés dans cet article partagent plusieurs aspects en commun: une ressource sensorielle et sémiotique comme le toucher, un contexte politique issu du féminisme et de l'activisme LGBTQ et la projection d'autres espacetemps dans lesquels sont proposés de nouveaux corps, de nouvelles relationnalités et des communautés qui ne cessent de se redessiner et se réajuster au cours des interactions. En tant que ressource esthétique et politique de connaissance de soi et du monde, le toucher se situe dans ce que j'ai appelé « une poétique et une politique de l'expérience ", dans lesquelles l'imagination et l'expérience jouent un rôle central pour penser à d'autres corporalités et à d'autres intersubjectivités possibles. Dans le cas des drag kings, on assiste à ce que j'ai appelé un dédoublement tactile corporel. Au cours de cette pratique, l'experte, tout en touchant son corps devant une novice qui le regarde, montre comment son visage pourrait être fabriqué et devenir. Dans ce cas-là, nous sommes face à une scène interactionnelle dans laquelle il y a un soi qui touche, une autre qui regarde et un tiers imaginé projeté dans un futur plus ou moins proche. Dans les autres exemples, issus des pratiques performatives et relevant des groupes de conscience, le toucher, tout en étant ancré dans l'expérience personnelle des sujets, excède les corps pour donner forme à de nouvelles philosophies cinématographiques, à de nouvelles relationnalités et affectivités dans le monde. En s'inspirant de ce dont Giovanna, l'une des personnes interviewées, m'avait fait part lors des entretiens, on peut dire que le toucher, dans cette dynamique d'ancrage corporel et de projection vers des réalités possibles excédant les existences, ici et maintenant, des sujets, permet de " faire monde " (fare mondo). "Faire monde » est une expression que Giovanna situe dans le vocabulaire féministe italien des années 70 . Elle désigne une manière particulière de voir et de vivre le monde de par sa propre présence et de par son corps. C'est dans ce cadre précisément que le toucher devient une ressource esthétique, un instrument de lutte politique et une ressource profondément queer. Il permet alors 
d'actualiser le projet féministe de faire de l'expérience corporelle, de sa perception et sensorialité, un moteur pour l'action politique et pour la création artistique, en faisant de soi et de l'autre, un monde imaginé et possible.

\section{BIBLIOGRAPHIE}

ALAIMO, Stacy. 2010. Bodily Natures. Science, Environment, and the Material Self, Bloomington: Indiana University Press.

ALAIMO, Stacy \& HEKMAN, Susan (eds.). 2008. Material Feminisms. Bloomington: Indiana University Press.

ALAIMO, Stacy \& HEKMAN, Susan. 2008a. « Introduction: Emerging Models of Materiality in Feminist Theory », in Material Feminisms. Bloomington: Indiana University Press : 1-22.

BARAD, Karen. 2003. « Posthumanist Performativity: Toward an Understanding of How Matter Comes to Matter » Signs: Journal of Women in Culture and Society 28-3: 801-831.

BARAD, Karen. 2012. « On Touching - The Inhuman that Therefore I Am » Differences: A Journal of Feminist Cultural Studies 23(3): 206-223.

BHABHA, Homi K. 2007 [1994]. Les lieux de la culture. Une théorie post-coloniale. Paris : Payot.

Boston Women's Health Book Collective. 1973. Our bodies, ourselves. Touchstone.

BRAIDOTTI, Rosi. 1991. Patterns of Dissonance: A Study of Women and Contemporary Philosophy. Cambridge: Polity Press.

BRAIDOTTI, Rosi. 2014. Il postumano. La vita oltre l'individuo oltre, la specie, oltre la morte. Roma : Derive \& Approdi.

BUCHOLTZ, Mary, HALL, Kira. 2016. « Embodied Sociolinguistics » Sociolinguistics. Theoretical Debates: 173-198.

BUTLER, Judith. 1993. Bodies that matter. New York: Routledge.

CHOULET, Anaïs. 2019. «Quand la philosophie touche aux questions de santé sexuelle. Retours sur une recherche pragmatique ancrée dans la militance féministe et la pratique thérapeutique », Intervention dans le cadre de la journée d'études doctorales « Une philosophie de terrain? » du 29 janvier 2019 [En ligne], consultée le 13 novembre 2019. URL : https://www.youtube.com/ watch?v=JYLvWyYg3-s

DE LANDA, Manuel. 2016. Assemblage Theory. Edinbourgh: Edinbourgh University Press.

DELEUZE, Gilles. 1991. Francis Bacon. La logique de la sensation. Paris : La différence.

DELEUZE, Gilles \& GUATTARI, Felix. 1980. Capitalisme et schizophrénie. Mille plateaux. Paris : Minuit.

DEWEY, John. 1934. L'art comme expérience. Paris : Folio Gallimard (édition 2010).

DODIER, Nicolas \& STAVRIANAKIS, Anthony (éds.). 2018. Les objets composés. Agencements, dispositifs, assemblages. Numéro spécial de Raisons Pratiques 28. 
DOLPHIJN, Rick \& van der TUIN, IRIS. 2012. New Materialism: Interviews and Cartographies. Ann Arbor: Open Humanity Press.

FOUCAULT, Michel. 1984. Histoire de la sexualité II. L'usage des plaisirs. Paris : Tel Gallimard.

GARFINKEL, Harold. 1967. Studies in Ethnomethodology. New Jersey: Prentice-Hall.

GIBSON, James. 1962. « Observations on Active Touch » Psychological Review 69-6: 477-491.

GOFFMAN, Erving. 1956. « The Nature of Deference and Demeanor » American Anthropologis 58:

473-502.

GOFFMAN, Erving. 1961. Encounters: Two studies in the sociology of interaction. Indianapolis: BobbsMerrill.

GOFFMAN, Erving. 1976. Gender Advertisements. New York: Harper and Row.

GOFFMAN, Erving. 1977. « The Arrangement between The Sexes » Theory and Society 4-3: 301-333.

GOODWIN, Charles. 2000. " Action and embodiment within situated human interaction » Journal of Pragmatics 32: 1489-1522.

GOODWIN, Charles. 2017. Co-operative Action. Cambridge: Cambridge University Press.

GOODWIN, Marjorie Harness \& CEKAITE, Asta. 2018. Embodied Family Choreography: Practices of Control, Care, and Mundane Creativity. New York: Routledge.

GRECO, Luca (éd.). 2014. "Les recherches linguistiques sur le genre : bilan et perspectives », Numéro spécial de Langage et Société 148.

GRECO, Luca (éd.). 2015. « Genre, langage et sexualité : données empiriques », Numéro spécial de Langage et Société 152.

GRECO, Luca. 2016. «Le travail de Charles Goodwin à l'épreuve de la performance » Tracés « Traduire et introduire » $3: 89-100$.

GRECO, Luca. 2017. «La performance au carrefour des arts et des sciences sociales : quelles questions pour la sociolinguistique ?» Langage et Société 140-141 : 301-317.

GRECO, Luca. 2018. Dans les coulisses du genre. La fabrique de soi chez les Drag Kings. Limoges : Lambert Lucas.

GRECO, Luca, GALATOLO, Renata, HORLACHER, Anne Sylvie, PICCOLI, Vanessa, TICCA, Anna Claudia \& URSI, Biagio. 2019. " Some theoretical and methodological challenges of transcribing touch in talk-in-interaction » Social Interaction. Video-Based Studies of Human Sociality [En ligne], 2 (1) consulté le 13 novembre 2019. URL : https://tidsskrift.dk/socialinteraction/article/view/ 113957.

HALL, Judith A. \& VECCIA, Ellen M. 1992. « Touch Asymmetry Between the Sexes » Gender Interaction and Inequality: 81-96.

HENLEY, Nancy. 1973. « Status and sex: Some touching observations » Bulletin of the Psychonomic Society 2: 91-93.

KAPROW, Allan. 1993. Essays on the blurring of Art and Life. Berkeley: University of California Press.

IRIGARAY, Luce. 1997. Être deux. Paris : Minuit.

LEVI, Corrado. 2010. « Méthodes et contenus des premières réunions du groupe FUORI ! de Milan : une histoire palpitante et violente » Genre, sexualité \& société [En ligne], 3, consulté le 23 janvier 2018. URL : http://journals.openedition.org/gss/1358 ; DOI : 10.4000/gss.1358. 
LEVINAS, Emmanuel. 1962. Totalité et infini. Paris : Livre de poche (édition 1990).

MACHIN, David, CALDAS-COULTHARD, Carmen Rosa, MILANI, Tommaso. 2016. « Doing critical multimodality in research on gender, language and discourse » Gender and Language 10-3: 301-308.

MANNING, Erin. 2007. Politics of Touch. Sense, Movement, Sovereignty. Minneapolis: Minnesota University Press.

MacKINNON, Catharine. 1982. « Feminism, Marxism, Method, and the State: An Agenda for Theory » Signs 7: 515-544.

MELANDRI, Lea. 2002. Una visceralita' indicibile. La pratica dell'inconscio nel movimento delle donne degli anni settanta. Milano: Franco Angeli.

MERLEAU-PONTY, Maurice. 1964. Le visible et l'invisible. Paris : Gallimard.

MONDADA, Lorenza. 2014. « The local constitution of multimodal resources for social interaction » Journal of Pragmatics 65: 137-156.

MONDADA, Lorenza. 2016. « Challenges of multimodality: Language and the body in social interaction » Journal of Sociolinguistics 20-3: 336-366.

MUPRHY, Keith. 2005. « Collaborative imagining: The interactive use of gestures, talk, and graphic representation in architectural practice » Semiotica 156: 113-145.

NISHIZAKA, Aug. 2003. « Imagination in Action » Theory and Psychology 13 (2): 177-207

PATERSON, Mark \& DODGE, Martin (eds.). 2012. Touching Space. Placing Touch. Surrey: Ashgate.

PUIG DE LA BELLACASA, María. 2009. « Touching technologies, touching visions.

The reclaiming of sensorial experience and the politics of speculative thinking " Subjectivity 28: 297-315.

SCHUSTERMAN, Richard. 2018. « Expérience esthétique et effrangement des frontières : Les aventures de l'homme en or » in À la frontière des arts. Lectures contemporaines de l'esthétique adornienne, LAFORGE, Wilfried \& LAGEIRA, Jacinto (éds.). Milan : Mimésis, 195-207.

STIER, Deborah S. \& HALL, Judith A. 1984. " Gender Differences in Touch: An Empirical and Theoretical Review » Journal of Personality and Social Psychology 47-2: 440-459.

TUANA, Nancy. 2008. « Viscous Porosity: Witnessing Katrina » Material Feminisms:188-213.

VASSELEU, Cathryn. 2015. « Resistances of Touch » Signs 40-2: 295-300.

ZANCARINI-FOURNEL, Michelle. 2004. " Notre corps, nous-mêmes » in Le siècle des feminismes, ZANCARINI-FOURNEL, Michelle, GUBIN, Eliane, JACQUES, Catherine, ROCHEFORT, Florence, STUDER, Brigitte \& THÉBAUD, Françoise (éds.), Paris : Les éditions de l'atelier, 209-220.

\section{NOTES}

1. Le terme d'assemblage est pour l'instant utilisé dans son sens commun tout en reconnaissant une dette importante envers la notion d'agencement proposée par Deleuze et Guattari (1980) et celle d'assemblage (De Landa 2016) dont la portée heuristique reste à vérifier pour nos données. Il se peut que d'ici quelques temps j'opte sur le plan terminologique pour un choix autour du terme d'agencements. Pour l'instant, je préfère le terme d'assemblage tout en sachant que cela pourra changer au fil du temps et en assumant une nécessaire phase de flottement sémantique entre les 
deux termes/positions. Pour une mise au point autour des notions d'assemblage, agencement et dispositifs, je renvoie à Dodier \& Stavrianakis (2018).

2. Il existe dans Greco (2018) une ébauche d'analyse sur une partie de cet extrait qui à l'époque n'avait pas mis au centre des analyses les pratiques tactiles comme des dispositifs de construction et de déconstruction du genre. C'est la raison pour laquelle, ici, on trouvera une analyse plus approfondie et portant sur une portion plus large de l'extrait.

3. Pour les conventions de transcription, je renvoie à :

http://icar.cnrs.fr/projets/corinte/documents/2013_Conv_ICOR_250313.pdf icar.cnrs.fr/projets/corinte/documents/convention_transcription_multimodale.pdf

4. D'autant plus qu'elle fait écho au projet esthétique et politique du pragmatisme : «L'objectif sous-jacent du pragmatisme était d'améliorer le projet démocratique et social visant à rendre l'art contemporain plus signifiant pour un public élargi, mais aussi construire un monde social plus satisfaisant d'un point de vue esthétique - en créant des relations harmonieuses et réciproquement enrichissantes entre les individus et les groupes sociaux » (Shusterman 2018 : 198).

5. https://www.youtube.com/watch?v=HGXEdqaEy18

\section{RÉSUMÉS}

Dans cet article, il s'agit d'analyser le rôle joué par le toucher dans la construction et la déconstruction du genre dans une multiplicité de contextes: ateliers drag king, groupes de conscience chez les féministes, et performances artistiques. Les enjeux de ce texte sont à la fois théoriques et analytiques. D'une part, il s'agit d'ouvrir un espace de dialogue entre les courants néo-matérialiste et postmoderne tels qu'il se déploient dans les études de genre et queer et les recherches sur le genre, le langage et la sexualité autour des imbrications possibles entre matérialité corporelle et discours. D'autre part, je verrai comment la prise en compte d'une approche multimodale du genre permet de saisir ce que j'appelle une poétique et d'une politique de l'expérience tactile excédant la temporalité et la spatialité des interactions et permettant de penser le genre au prisme de la sensorialité, de l'expérience et de l'imagination.

Drawing from a multiplicity of contexts (drag king workshops, consciousness raising groups and artistic performances), I will analyze how touching practices are mobilized and interpreted by social actors as gender (de)construction devices. The issues presented in this paper are both theoretical and analytical. I will propose a theoretical dialogue between two antagonistic traditions in feminism, neo-materialism and postmodernism, and gender, language and sexuality studies around the possible intertwinings between discourse and bodily materiality. I will show how a multimodal approach to gender takes into account what I call a poetic and a politics of tactile experience exceeding the temporality and the spatiality of interactions and to think about gender through the lens of sensoriality, experience and imagination. 
INDEX

Thèmes : Recherches

Mots-clés : toucher, expérience, imagination, multimodalité, queer, néo-matérialisme

Keywords : touching, experience, imagination, multimodality, queer, neo-materialism

\section{AUTEUR}

LUCA GRECO

Luca Greco est professeur de sociolinguistique à l'Université de Lorraine, Metz. Yel développe depuis plusieurs années une linguistique profondément ancrée dans les sciences sociales et irréductiblement transdisciplinaire. Elle croise l'anthropologie linguistique avec les recherches sur la multimodalité, les études de genre et les Performance Studies. Ses centres d'intérêt se situent autour des pratiques de catégorisation dans la parole, les textes et les corps en interaction, des relations entre genre, sexualité et langage et de l'étude de la performance dans les arts, les sciences sociales et les pratiques quotidiennes. Yel est rédacteur en chef de la revue Langage et Société. 